\title{
Ausbildungsvergütungen in Deutschland als Ausbildungsbeihilfe oder Arbeitsentgelt
}

\author{
Der Gesetzgeber hat Ende der 1960er Jahre mit dem Berufsbildungsgesetz den Ausbil- \\ dungsvergütungen sowohl die Funktion einer Beihilfe zum Lebensunterhalt der Auszubil- \\ denden als auch die einer Entlohnung für erbrachte Arbeitsleistungen zugeschrieben. \\ Steht eine dieser Funktionen heute bei der Festlegung der Vergütungshöhe im Vorder- \\ grund oder haben Beihilfe- und Entgeltfunktion gleichermaßen Bedeutung? Um diese \\ Kernfrage geht es in folgendem Beitrag. Dabei wird auf empirischer Grundlage unter- \\ sucht, inwieweit sich die Vergütungsfestlegung in der Praxis an den produktiven Leistun- \\ gen der Auszubildenden im Betrieb orientiert und inwieweit die Vergütungszahlungen als \\ Beihilfe aufgefasst werden, deren Höhe durch soziale Aushandlungsprozesse bestimmt \\ wird.
}

URSULA BEICHT, GÜNTER WALDEN

\section{Einführung}

In Deutschland haben Auszubildende gegenüber ihren Ausbildungsbetrieben einen rechtlichen Anspruch auf eine angemessene Vergütung ( $\$ 17$ Berufsbildungsgesetz). Der Gesetzgeber hat den Ausbildungsvergütungen seinerzeit sowohl die Funktion einer Beihilfe zum Lebensunterhalt der Auszubildenden als auch die einer Entlohnung für erbrachte Arbeitsleistungen zugeschrieben (Deutscher Bundestag 1969). Dies war ein Kompromiss angesichts einer lang anhaltenden Diskussion um das Wesen der Ausbildungsvergütungen (Beicht 2011). Während die Gewerkschaften seit Anfang des 20. Jahrhunderts die Vergütung der Auszubildenden als Arbeitslohn ansahen, wurde sie vor allem vom Handwerk als Beihilfe aufgefasst. Im Folgenden wird der Frage nachgegangen, von welchen Faktoren die Höhe der Vergütungen heute bestimmt wird und ob dabei eher der Entlohnungs- oder eher der Beihilfecharakter der Ausbildungsvergütungen eine Rolle spielt. Da die angemessene Höhe der Ausbildungsvergütungen zwischen Arbeitgebern und Gewerkschaften häufig kontrovers diskutiert wird, ist es wichtig, die Grundlagen der Vergütungsfestsetzung zu klären. Angesichts der gleichzeitigen Zugehörigkeit von Auszubildenden zum Ausbildungs- und zum Beschäftigungssystem gibt dies auch Aufschluss darüber, ob die Vergütungen eher zum Ausbildungscharakter (Beihilfe) oder zum Beschäftigungscharakter der Ausbildung (Entlohnung) passen.
Kennzeichen einer Ausbildungsbeihilfe ist es, dass sie bedarfsorientiert festgelegt wird. In ihrer strengsten Form wäre eine solche Beihilfe für alle Auszubildenden weitestgehend gleich hoch. Eine Variation ließe sich dann nur über Unterschiede im individuellen Bedarf rechtfertigen. Faktisch unterscheiden sich die Ausbildungsvergütungen in Deutschland zwischen Wirtschaftszweigen und Berufen jedoch so erheblich (Beicht 2011; Hans-Böckler-Stiftung 2012), dass sie keinesfalls eine Ausbildungsbeihilfe in diesem strengen Sinne darstellen können. Dies bedeutet nun aber nicht zwangsläufig, dass Ausbildungsvergütungen im Wesentlichen die Funktion einer Entlohnung für erbrachte Arbeitsleistungen haben. Vielmehr könnte es auch sein, dass der Bedarf der Auszubildenden nicht als fester absoluter Wert, sondern als relative Größe in Abhängigkeit davon definiert wird, was in einem Wirtschaftszweig oder einem Betrieb als angemessen gilt. Die Orientierungsgröße dafür könnte das durchschnittliche Lohnniveau der Beschäftigten sein, das sich ebenfalls zwischen Wirtschaftszweigen und Betrieben deutlich voneinander unterscheidet. Ausbildungsvergütungen hätten auch bei einer solchen relativen Bedarfsbestimmung eher den Charakter einer Ausbildungsbeihilfe und nicht den eines Arbeitsentgelts. Die Ausbildungsvergütung als Entgelt für erbrachte Arbeitsleistungen würde dagegen implizieren, dass die Vergütungshöhe wesentlich durch den Produktivbeitrag der Auszubildenden bestimmt würde. 
Im Rahmen einer empirischen Analyse wird im Folgenden untersucht, worauf Unterschiede in den gezahlten Ausbildungsvergütungen zurückgeführt werden können und ob sich hieraus Hinweise für den Charakter von Ausbildungsvergütungen als einer Ausbildungsbeihilfe oder eines Arbeitsentgeltes ableiten lassen. Hinsichtlich des Charakters eines Arbeitsentgeltes wird der Produktivbeitrag der Auszubildenden betrachtet. Hinsichtlich des Charakters einer Ausbildungsbeihilfe werden mögliche tarifrelevante und betriebliche Referenzgrößen herangezogen. Dabei wird von folgenden Überlegungen ausgegangen: Überbetrieblich wird der Bedarf der Auszubildenden über kollektive Aushandlungsprozesse im Rahmen von Tarifverhandlungen bestimmt. Sofern sich die dort festgelegten Ausbildungsvergütungen am Lohnniveau des Tarifbereiches orientieren, würde dies zu Vergütungsunterschieden zwischen Wirtschaftszweigen, Regionen, Ausbildungsbereichen und Betriebsgrößenklassen führen. Auf der Ebene des einzelnen Betriebs würde das durchschnittliche Lohnniveau seiner Beschäftigten den Orientierungsmaßstab für den Bedarf der Auszubildenden bilden und damit ebenfalls die Höhe der Ausbildungsvergütungen beeinflussen. Zusätzlich müssen dabei allerdings noch weitere betriebliche Faktoren berücksichtigt werden. So ist es von Relevanz, inwieweit überbetriebliche Sachverhalte über die kollektivvertraglichen Vereinbarungen auf der betrieblichen Ebene tatsächlich Anwendung finden. In diesem Zusammenhang ist insbesondere die Tarifgebundenheit des Betriebs zu betrachten. Zudem könnte es auch noch einen Einfluss auf die Vergütungshöhe haben, aus welchem Motiv heraus sich der Betrieb für die eigene Ausbildung entschieden hat.

Dieser Beitrag ist wie folgt aufgebaut: Zunächst werden grundlegende methodische Hinweise zur verwendeten $\mathrm{Da}$ tenbasis und zur Vorgehensweise gegeben (Abschnitt 2). Abschnitt 3 zeigt die Unterschiede in der Höhe der Ausbildungsvergütungen in Deutschland auf. Abschnitt 4 enthält die empirischen Ergebnisse zu den betrieblichen Rahmenbedingungen der Vergütungszahlungen. In Abschnitt 5 wird der Umfang der produktiven Leistungen der Auszubildenden beschrieben und in Abschnitt 6 folgt eine Darstellung der Ausbildungsvergütungen in Relation zum Lohn- und Gehaltsniveau. In Abschnitt 7 wird auf der Grundlage eines statistischen Modells das relative Gewicht der verschiedenen möglichen Einflussfaktoren betrachtet. Der Beitrag schließt mit einer kurzen Zusammenfassung und Schlussfolgerungen (8).

\section{Methodische Hinweise}

Die Basis der Analysen bilden die Daten der vierten KostenNutzen-Erhebung des Bundesinstituts für Berufsbildung (BIBB) zur betrieblichen Berufsausbildung (Schönfeld et al. 2010). Dabei handelt es sich um eine im Jahr 2008 durchgeführte repräsentative Befragung von Ausbildungsbetrieben. Zu diesem Zweck ist eine Zufallsstichprobe von knapp
8.500 Betrieben (bereinigte Bruttostichprobe) aus der Betriebsdatei der Bundesagentur für Arbeit (BA) gezogen worden. Diese umfasst alle deutschen Betriebsstätten mit mindestens einem sozialversicherungspflichtig Beschäftigten. Berücksichtigt wurden Betriebe, die in einem von 51 ausgewählten stark besetzten Berufen ausbildeten. Die Befragung erfolgte mittels computergestützter persönlicher Interviews (CAPI) für das Bezugsjahr 2007. Nach Ende der Erhebungsphase lagen 2.986 auswertbare Interviews (Nettostichprobe) vor. Die Rücklaufquote lag damit bei $35 \%$. Die Wahl des Datensatzes ergibt sich daraus, dass allein er die erforderlichen Variablen zur Höhe der Vergütungen und zu den Produktivleistungen der Auszubildenden abbildet. Neuere Daten liegen gegenwärtig nicht vor. Die im Aufsatz behandelte Fragestellung wurde bisher noch nicht untersucht.

Um zu repräsentativen Ergebnissen bezogen auf alle Auszubildenden des Jahres 2007 zu kommen, wurde der Datensatz mittels Gewichtung an die Auszubildendenstrukturen in der Grundgesamtheit angepasst und hochgerechnet (Schönfeld et al. 2010, S. 30f.). Zugrunde gelegt wurden dabei die aus der Berufsbildungsstatistik des Statistischen Bundesamtes und der Beschäftigtenstatistik der BA bekannten Verteilungen der Auszubildenden nach Beruf, Ausbildungsbereich, Ausbildungsjahr, Betriebsgrößenklasse sowie West- und Ostdeutschland. Des Weiteren wurde eine alternative Gewichtung auf Betriebsebene vorgenommen. Hierfür wurde die aus der BA-Beschäftigtenstatistik vorliegende Gesamtverteilung der Betriebe nach Betriebsgröße und Wirtschaftszweigen herangezogen. Damit ist es auch möglich, repräsentative Ergebnisse bezogen auf alle Ausbildungsbetriebe zu ermitteln.

Für die im Folgenden dargestellten Analysen wurden beide Gewichtungen herangezogen. Die gebildeten Durchschnitte, z. B. der Ausbildungsvergütungen, basieren ausschließlich auf dem anhand der Auszubildendenstrukturen gewichteten Datensatz, es handelt sich also immer um durchschnittliche Werte pro Auszubildenden. Verteilungen, z. B. die Häufigkeit einer tariflichen Bindung bei den Ausbildungsvergütungen, werden sowohl für die Betriebe als auch für die Auszubildenden ausgewiesen, berechnet auf Grundlage der jeweils entsprechend gewichteten Datensätze. Für die multivariaten Analysen wurde hingegen ein ungewichteter Datensatz verwendet.

Bei einer Analyse der Ausbildungsvergütungen auf Basis der BIBB-Kosten-Nutzen-Erhebung besteht die Schwierigkeit, dass die einzelnen befragten Betriebe im Bezugsjahr häufig nicht in allen Ausbildungsjahren ausbildeten. Für viele Betriebe liegen daher nur für bestimmte Ausbildungsjahre die Vergütungsangaben vor. Da sich die Vergütungen in den einzelnen Ausbildungsjahren erheblich unterscheiden, ist eine Durchschnittsbildung pro Betrieb nicht

\footnotetext{
Die Autoren möchten an dieser Stelle der Projektgruppe der BIBB-Kosten-Nutzen-Erhebung 2007 für die Bereitstellung der Erhebungsdaten sowie der zentralen Kosten-Nut-
} zen-Variablen vielmals danken. 
sinnvoll, sofern die Angaben nicht vollständig vorliegen. ${ }^{2}$ Daher erfolgen die Analysen zum Zusammenhang von Ausbildungsvergütungen und Arbeitskraftentgelten bzw. produktiven Leistungen der Auszubildenden exemplarisch zum einen für das erste und zum anderen für das dritte Ausbildungsjahr. Dabei sind jeweils ausschließlich die Betriebe einbezogen, die im Bezugsjahr 2007 Auszubildende im ersten bzw. im dritten Ausbildungsjahr hatten.

\section{Vergütungsunterschiede}

Die Vergütungen, die die Betriebe ihren Auszubildenden zahlen, variieren in Deutschland sehr stark. So steigen die Vergütungsbeträge gemäß der gesetzlichen Regelung in $\$ 17$ Berufsbildungsgesetz „mit fortschreitender Berufsausbildung, mindestens jährlich“ an, d.h. in der Praxis erhöhen sie sich in der Regel von Ausbildungsjahr zu Ausbildungsjahr. Für die Betriebe und Auszubildenden viel bedeutender sind allerdings die Unterschiede im Vergütungsniveau, die sich zwischen den Wirtschaftszweigen und Regionen herausgebildet haben.

Die tarifvertraglichen Vereinbarungen haben eine zentrale Bedeutung für die jeweilige Höhe der gezahlten Ausbildungsvergütungen. In den für die einzelnen Wirtschaftszweige und Regionen durchgeführten Tarifverhandlungen befinden die Arbeitgeber- und Arbeitnehmervertreter darüber, welche Beträge für ihren Tarifbereich jeweils eine „angemessene" Ausbildungsvergütung darstellen. Dabei haben sie nach dem Willen des Gesetzgebers, wie eingangs gesagt, sowohl die Beihilfe- als auch die Entgeltfunktion der Vergütungszahlungen zu beachten (Deutscher Bundestag 1969). So soll zum einen eine fühlbare finanzielle Unterstützung des Lebensunterhalts, zum anderen eine Mindestentlohnung für die im Betrieb geleistete produktive Arbeit des Auszu- bildenden gewährleistet sein. Im Ergebnis fallen die tariflichen Ausbildungsvergütungen in den Wirtschaftszweigen und Regionen und damit verknüpft zwischen den Ausbildungsbereichen und Betriebsgrößen äußerst unterschiedlich aus (Beicht 2011; Beicht/Krewerth 2010; Hans-BöcklerStiftung 2012). Zudem differiert die Vergütungshöhe zwischen kaufmännischen und gewerblichen bzw. technischen Berufen. Die Unterschiede in den tariflichen Ausbildungsvergütungen dürften zum einen auf unterschiedliche wirtschaftliche Ausgangsbedingungen in den Tarifbereichen zurückzuführen sein. Zum anderen dürfte aber auch eine Rolle spielen, welches Gewicht die Gewerkschaften bei der Gestaltung von Tarifverträgen der Ausbildung im Vergleich $\mathrm{zu}$ anderen $\mathrm{zu}$ regelnden Inhalten zumessen.

Nach der BIBB-Kosten-Nutzen-Erhebung 2007 liegen die monatlichen Bruttoausbildungsvergütungen im Gesamtdurchschnitt pro Auszubildenden bei $605 €^{3}$ Im ersten Ausbildungsjahr betragen sie durchschnittlich $535 €$, im zweiten $598 €$, im dritten $664 €$ und im vierten Jahr $713 €$. Nach den einzelnen Strukturmerkmalen gibt es sehr starke Abweichungen. So erhalten z.B. im ersten Ausbildungsjahr die Auszubildenden in Westdeutschland mit durchschnittlich $564 €$ erheblich höhere Vergütungen als diejenigen in Ostdeutschland mit $430 €$. Im Hinblick auf die Wirtschaftszweige ${ }^{\circledR}$ werden im Dienstleistungsbereich I, zu dem unter anderem das Gastgewerbe zählt, die Auszubildenden im ersten Jahr mit $491 €$ deutlich schlechter bezahlt als im Dienstleistungsbereich II (627 €), dem unter anderem das Kredit- und Versicherungsgewerbe zuzurechnen ist. Mit zunehmender Größe der Ausbildungsbetriebe steigen die Vergütungsbeträge deutlich an. In den Ausbildungsbereichen Öffentlicher Dienst sowie Industrie und Handel ist die Vergütungssituation für die Auszubildenden wesentlich günstiger als im Handwerk, der Landwirtschaft und bei den Freien Berufen. Die Auszubildenden in kaufmännischen Berufen kommen auf höhere Vergütungen als diejenigen in gewerblichen bzw. technischen Berufen. ${ }^{\oplus}$
(2) Es hätte zwar die Möglichkeit bestanden, durch Imputationen die Vergütungsangaben auch für die nicht besetzten Ausbildungsjahre zu vervollständigen. Da dieses Verfahren jedoch bedeutet, dass Schätzwerte für faktisch nicht vorhandene Auszubildende eingesetzt werden, wurde hier darauf verzichtet und ausschließlich auf die Daten der tatsächlich besetzten Ausbildungsjahre zurückgegriffen.

(3) Nach Berechnungen des BIBB ist für 2007 ein Gesamtdurchschnitt der tariflichen Ausbildungsvergütungen von $628 €$ pro Monat zu verzeichnen (Beicht 2011). Der im Vergleich dazu niedrigere Wert, der sich auf Basis der BIBBKosten-Nutzen-Erhebung 2007 ergibt, dürfte vor allem auf die Einbeziehung nicht tariflicher Vergütungszahlungen zurückzuführen sein. Aktuell, d. h. für 2011 errechnete das BIBB einen Durchschnitt der tariflichen Ausbildungsvergütungen von $700 €$ pro Monat (BIBB 2012).

(4) Es handelt sich hierbei um eine sehr starke Zusammenfassung der Wirtschaftszweige in lediglich sechs Gruppen.
5 Wir bezeichnen Wirtschaftszweig, Region, Ausbildungsbereich und Betriebsgröße (klassische betriebliche Variablen) als tarifrelevante Betriebsmerkmale und zwar aus folgendem Grund: Die tariflichen Vereinbarungen spiegeln die auf der kollektivvertraglichen Ebene vorhandenen Vorstellungen zur angemessenen Ausbildungsvergütung wider. Die fachlichen und regionalen Geltungsbereiche der Tarifverträge sind allerdings so differenziert, dass die Varianz der Vergütungsvereinbarungen bei Zusammenfassung in sechs Wirtschaftszweiggruppen nicht mehr genügend zum Ausdruck kommt. Die Tatsache, dass sich die Vereinbarungen auch zwischen den Kammerbereichen (z.B. Industrie- und Handel/Handwerk), Regionen (Ost/West), Tarifbereichen mit klein- bzw. großbetrieblichen Strukturen, kaufmännischen und gewerblich-technischen Berufen unterscheiden, wurde daher über die Variablen Ausbildungsbereich, Region, Betriebsgrößenklasse, Berufsart berücksichtigt. Sie stehen für Merkmale der jeweiligen Tarifbereiche und kennzeichnen die kollektivvertraglichen Vereinbarungen zu den Ausbildungsvergütungen. 
Die tatsächlich gezahlten Ausbildungsvergütungen entsprechen bei Weitem nicht immer den tariflichen Sätzen. Vielmehr kann die Vergütungshöhe auch von bestimmten betrieblichen Rahmenbedingungen beeinflusst werden, auf die nun kurz eingegangen werden soll.

\section{Betriebliche Rahmenbedingungen der Vergütungszahlungen}

Die Höhe der Vergütungszahlungen an die Auszubildenden hängt nicht nur von dem Wirtschaftszweig und der Region $\mathrm{ab}$, in dem der Betrieb angesiedelt ist, sondern auch davon, ob für ihn eine Tarifbindung bei den Ausbildungsvergütungen besteht oder nicht. Tarifgebundene Betriebe ${ }^{\bullet}$ müssen mindestens die für ihren Bereich tariflich festgelegten Sätze zahlen, sie haben daher bei ihren Vergütungszahlungen nur einen Flexibilitätsspielraum nach oben. Betriebe, die keinem Tarifvertrag unterliegen, haben bei den Ausbildungsvergütungen größere Freiheitsgrade. Ihnen steht nicht nur frei, die Tarife ihres Wirtschaftszweigs und ihrer Region zu überschreiten, sondern sie dürfen nach geltender Rechtsprechung auch um bis zu 20 \% darunter bleiben (Herkert/Töltl 2010). Damit ist anzunehmen, dass die tatsächlich gezahlten Vergütungen in nicht tarifgebundenen Betrieben tendenziell niedriger ausfallen als in tarifgebundenen Betrieben.

Die Anteile der Betriebe, die an eine tarifliche Regelung der Ausbildungsvergütungen gebunden sind, unterscheiden sich sehr stark nach den verschiedenen Strukturmerkmalen. Insgesamt unterliegen 2007 etwas mehr als die Hälfte der Betriebe (54,2 \%) einem Tarif, in ihnen sind knapp zwei Drittel der Auszubildenden (64,5 \%) vertreten. In Ostdeutschland ist eine Tarifbindung weit weniger verbreitet als in Westdeutschland (Tabelle 1). ${ }^{\circledR}$ Sehr selten sind die Betriebe im Dienstleistungsbereich II tarifgebunden, relativ oft hingegen im Verarbeitenden Gewerbe. Mit zunehmender Betriebsgröße steigt der Anteil tarifgebundener Betriebe deutlich an. Im Handwerk und vor allem in der Landwirtschaft kommt eine Tarifbindung bei den Ausbildungsvergütungen häufiger vor als in den Ausbildungsbereichen Industrie und Handel sowie Freie Berufe. ${ }^{\circ}$

Insgesamt gut ein Viertel der Ausbildungsbetriebe (27\%) ist zwar nicht tarifgebunden, zahlt die Ausbildungsvergütung aber dennoch in Anlehnung an einen Tarif, dies betrifft $22 \%$ der Auszubildenden. Immerhin knapp ein Fünftel der Betriebe (18,6 \%) - mit 13,3\% der Auszubildenden - orientiert sich nicht an einer tariflichen Regelung, ihre Vergütungszahlungen dürften somit vielfach untertariflich sein. In Ostdeutschland liegt der Anteil der betreffenden Betriebe fast doppelt so hoch wie in Westdeutschland.

Die Vergütungshöhe wird darüber hinaus möglicherweise auch davon beeinflusst, ob es im Ausbildungsbetrieb einen Betriebsrat gibt. Betriebsräte kümmern sich oft - gegebenenfalls gemeinsam mit der betrieblichen Jugend- und Auszubildendenvertretung - intensiv um die Belange der Auszubildenden im Unternehmen und achten dabei unter anderem auch auf eine tarifgemäße bzw. angemessene Bezahlung. ${ }^{\ominus}$ Betriebe mit einem Betriebsrat stehen möglicherweise auch Vorstellungen von einer sozialen Verantwortung von Unternehmen aufgeschlossener gegenüber. So ist zu erwarten, dass die Ausbildungsvergütungen in Betrieben mit einem Betriebsrat eher höher liegen als in Betrieben ohne einen Betriebsrat.

Nur in $13 \%$ der Ausbildungsbetriebe existiert 2007 ein Betriebsrat. In diesen Betrieben sind allerdings gut $42 \%$ der Auszubildenden anzutreffen, da das Vorhandensein eines Betriebsrats mit zunehmender Betriebsgröße stark ansteigt (Tabelle 1). Während sehr kleine Betriebe nur äußerst
(6) Eine Tarifbindung liegt in der Regel vor, wenn ein Betrieb dem Arbeitgeberverband angehört, der den in der jeweiligen Branche und Region geltenden Flächentarifvertrag abgeschlossen hat, oder wenn der betreffende Tarifvertrag für allgemeinverbindlich erklärt wurde; einige Unternehmen schließen auch eigene Firmentarifverträge ab. Zudem muss eigentlich auch der Auszubildende Mitglied der tarifschließenden Gewerkschaft sein, was jedoch in der Praxis keine Rolle spielt (Schnabel 2005).

(7) Nach den Ergebnissen des IAB-Betriebspanels 2007 ist die Tarifbindung bezogen auf alle Betriebe mit mindestens einem sozialversicherungspflichtig Beschäftigten allerdings erheblich geringer gewesen (Ellguth/Kohaut 2008). Danach sind $2007 \mathrm{im}$ Westen $39 \%$ der Betriebe und im Osten $24 \%$ tarifgebunden, $42 \%$ (West) bzw. $41 \%$ (Ost) lehnen sich an einen Tarif an und $20 \%$ (West) bzw. $35 \%$ (Ost) haben keinen Bezug zu einem Tarifvertrag. Die Abweichungen zu den Ergebnissen der BIBB-Kosten-Nutzen-Erhebung 2007 sind vor allem damit zu erklären, dass dieTarifbindung mit der Größe des Betriebs generell stark zunimmt (Bispinck/Schul- ten 2009) und bei Ausbildungsbetrieben größere Betriebe deutlich stärker vertreten sind als bei den Betrieben insgesamt. Zudem gibt es in manchen Bereichen zwar tarifliche Regelungen zu den Arbeitsbedingungen und Löhnen, aber nicht zu den Ausbildungsvergütungen.

(8) Der zunächst überraschend niedrig erscheinende Anteil im Ausbildungsbereich Industrie und Handel dürfte unter anderem darauf zurückzuführen sein, dass hier in vielen kleineren Betrieben des Dienstleistungsbereichs (z. B. in der IT-, Medien- und Veranstaltungsbranche) keine Tarifbindung besteht. Der ebenfalls relativ geringe Anteil im Ausbildungsbereich Öffentlicher Dienst hängt unter anderem damit zusammen, dass der dort zugehörige Beruf „Vermessungstechniker/-in" häufig nicht in der öffentlichen Verwaltung, sondern in Ingenieur- oder Vermessungsbüros ausgebildet wird, die meist nicht tarifgebunden sind.

(9 In tarifgebundenen Betrieben ist es unter anderem Aufgabe des Betriebsrats, die Umsetzung der geltenden Tarifverträge zu überwachen (Ellguth/Kohaut 2011). 
TABELLE 1

Betriebliche Rahmenbedingungen und Vergütungszahlungen

Anteil der Betriebe und Auszubildenden in Prozent (Zeilenprozente)

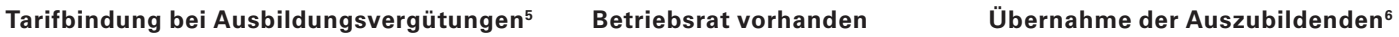

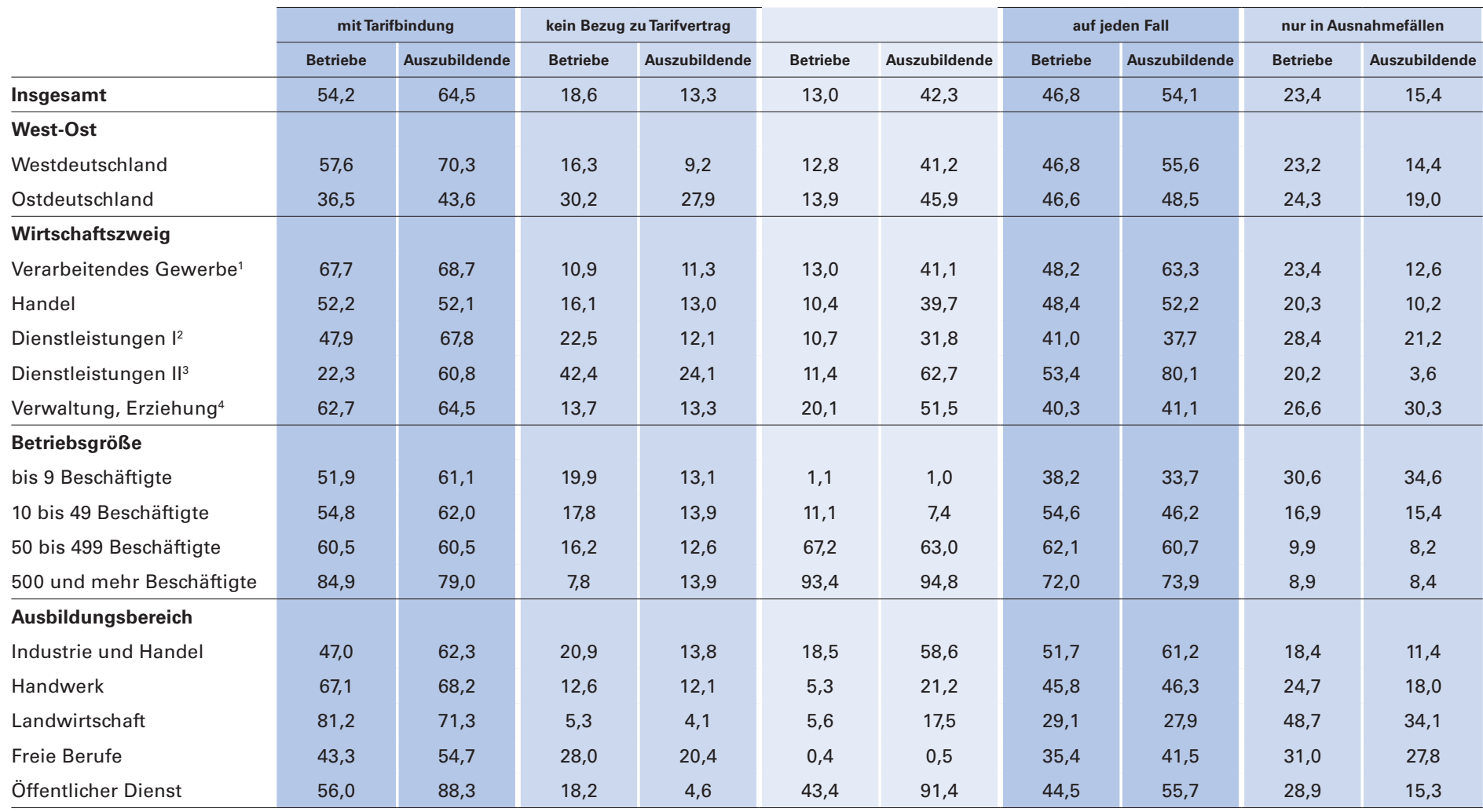

1 einschließlich Landwirtschaft;

${ }^{2}$ Gastgewerbe, Erbringung sonstiger öffentlicher und persönlicher Dienstleistungen, Verkehr und Nachrichtenübermittlung, Energie- und Wasserversorgung

${ }^{3}$ Kredit- und Versicherungsgewerbe, Grundstücks- und Wohnungswesen, Vermietung beweglicher Sachen, Erbringung von wirtschaftlichen Dienstleistungen

${ }^{4}$ Öffentliche Verwaltung, Verteidigung, Sozialversicherung, Erziehung und Unterricht, Gesundheits-, Veterinär- und Sozialwesen;

${ }^{5}$ nicht ausgewiesen ist der Anteil der Betriebe, die die Vergütung in Anlehnung an einen Tarif zahlen;

${ }^{6}$ nicht ausgewiesen ist der Anteil der Betriebe, die einen Teil ihrer Auszubildenden übernehmen wollen.

Quelle: BIBB-Kosten-Nutzen-Erhebung 2007.

selten einen Betriebsrat haben, sind es bei sehr großen Betrieben über $90 \%$. Sehr stark verbreitet sind Betriebsräte im Ausbildungsbereich Öffentlicher Dienst, relativ stark auch in Industrie und Handel. Im Bereich der Freien Berufe haben Betriebsräte dagegen Seltenheitswert.

$\mathrm{Ob}$ und inwieweit Betriebe ihren Flexibilitätsspielraum bei den Vergütungszahlungen nutzen, dürfte vor allem davon abhängen, welche Ausbildungsmotive für sie ausschlaggebend sind. Es lassen sich im Wesentlichen zwei Gründe für die eigene Durchführung von Ausbildung unterscheiden (vgl. Überblick in Schönfeld et al. 2010, S. 14ff.). Bei einem Produktionsmotiv stehen für den Betrieb die wirtschaftlich verwertbaren Arbeiten des Auszubildenden im Vordergrund und eine Übernahme nach der Ausbildung ist nicht geplant. Bei einem Investitionsmotiv geht es dem Betrieb um die Gewinnung von geeigneten Fachkräften unabhängig vom externen Arbeitsmarkt, die Übernahme der Auszubildenden ist vorgesehen. ${ }^{(0)} \mathrm{Da}$ sich bei einem Produktionsmotiv die Ausbildung nur dann lohnt, wenn die produktiven
Leistungen der Auszubildenden höher als die Kosten der Ausbildung sind, werden diese Betriebe die Ausbildungskosten möglichst gering halten und somit eher niedrige Vergütungen zahlen. Betriebe mit einem Investitionsmotiv wollen hingegen die Auszubildenden dauerhaft im Betrieb halten und werden daher eher bereit sein, sie bereits während der Ausbildung besser zu entlohnen. Wenzelmann (2012) hat in diesem Zusammenhang festgestellt, dass bei Betrieben mit einem Produktionsmotiv die produktiven Zeiten der Auszubildenden höher und bei Betrieben mit einem Investitionsmotiv niedriger sind.

Im Jahr 2007 hat insgesamt knapp die Hälfte der Betriebe (47\%) vor, ihre Auszubildenden auf jeden Fall zu übernehmen, bei ihnen liegt offensichtlich ein Investitionsmotiv

(10) Zum Produktionsmotiv vgl. Lindley 1975; zum Investitionsmotiv vgl. Merrilees 1983. 
bei der Ausbildung vor. ${ }^{(1)}$ Besonders häufig trifft dies auf größere Betriebe zu (Tabelle 1). Knapp ein Viertel der Betriebe (23\%) erwägt eine Weiterbeschäftigung von Auszubildenden nach Ausbildungsabschluss nur in Ausnahmefällen, bei ihnen dürfte meist ein Produktionsmotiv im Vordergrund stehen. Überdurchschnittlich oft kommt dies bei sehr kleinen Betrieben sowie Betrieben der Ausbildungsbereiche Landwirtschaft und Freie Berufe vor. Insgesamt 30 \% der Betriebe planen die Übernahme eines Teils ihrer Auszubildenden, hier spielen wahrscheinlich beide Ausbildungsmotive gleichzeitig eine Rolle.

Nachdem die betrieblichen Rahmenbedingungen aufgezeigt wurden, die möglicherweise die von den Betrieben gezahlten Ausbildungsvergütungen beeinflussen, soll im folgenden Abschnitt der Frage nachgegangen werden, in welchem Umfang die Auszubildenden in ihren Betrieben produktiv tätig sind und ob sich dabei erste Hinweise auf einen Zusammenhang mit der Höhe der Vergütungszahlungen finden lassen.

\section{Produktive Leistungen der Auszubildenden}

Bei Ermittlung der produktiven Leistungen von Auszubildenden ist zu beachten, dass zum einen die Schwierigkeit der verrichteten Tätigkeiten im Ausbildungsverlauf unterschiedlich ist und zum anderen auch der von den Auszubildenden erreichte Leistungsgrad variiert. In der BIBBKosten-Nutzen-Erhebung 2007 ist daher zwischen einfacheren Arbeiten unterschieden worden, die normalerweise von an- und ungelernten Arbeitskräften erledigt werden (produktive Tätigkeiten I), und schwierigen Aufgaben, die in der Regel von Fachkräften wahrgenommen werden (produktive Tätigkeiten II). Für beide Tätigkeitsarten ist die Zeitdauer zu erfassen, in der die Auszubildenden mit entsprechenden Arbeiten beschäftigt sind (produktive Tage I und II). Bei den produktiven Tätigkeiten I wird unterstellt, dass Auszubildende hierbei bereits ebenso gut und schnell wie an- und ungelernte Arbeitskräfte sind (Leistungsgrad $=100 \%$ ). Bei den Tätigkeiten II ist dagegen der in der Regel geringere Leistungsgrad der Auszubildenden im Vergleich zu einer Fachkraft noch zusätzlich zu ermitteln. Mit diesem Leistungsgrad werden die produktiven Tage II bewertet, im Ergebnis erhält man die Anzahl der Tage, die eine Fachkraft für die gleiche Arbeitsleistung benötigen würde (äquivalente Tage II).

Im Durchschnitt kommen 2007 die Auszubildenden im ersten Ausbildungsjahr auf 59 produktive Tage I (An- und Ungelerntentätigkeiten) und 19 äquivalente Tage II (Fachkraftleistungen). Im dritten Ausbildungsjahr gehen die Anund Ungelerntentätigkeiten auf 40 Tage zurück, während die Fachkraftleistungen auf 59 Tage ansteigen. Berücksichtigt sind dabei ausschließlich die produktiven Tätigkeiten, die am normalen betrieblichen Arbeitsplatz verrichtet werden, nicht jedoch eventuelle produktive Arbeiten in der betrieblichen Lehrwerkstatt. Wie aus Tabelle 2 hervorgeht, ist der produktive Einsatz der Auszubildenden in westdeutschen Betrieben insgesamt etwas höher als in ostdeutschen Betrieben. Bei den Wirtschaftszweigen fällt vor allem der relativ geringe Umfang der fachkraftgleichen Tätigkeiten im Verarbeitenden Gewerbe auf. In sehr kleinen Betrieben werden Auszubildende überdurchschnittlich oft für beide Arten produktiver Arbeiten herangezogen. In sehr großen Betrieben fallen die produktiven Zeiten am Arbeitsplatz insgesamt eher gering aus, was vor allem im ersten Ausbildungsjahr zum Teil mit der häufigeren Lehrwerkstattausbildung im gewerblich-technischen Bereich zusammenhängen dürfte. Besonders viel werden Auszubildende bei den Freien Berufen produktiv eingesetzt, vor allem im dritten Ausbildungsjahr sind hier die Fachkraftleistungen außerordentlich hoch. In der Landwirtschaft verrichten die Auszubildenden überdurchschnittlich häufig Tätigkeiten von An- und Ungelernten. Im Ausbildungsbereich Öffentlicher Dienst ist insgesamt ein eher geringer produktiver Einsatz zu verzeichnen.

Für den Betrieb sind die produktiven Arbeiten der Auszubildenden so viel wert, wie er alternativ einer an- oder ungelernten Arbeitskraft bzw. einer Fachkraft für die gleichen Leistungen zahlen müsste. Durch den Einsatz der Auszubildenden spart der Betrieb diese Lohnzahlungen ein, was daher als Ausbildungserträge bezeichnet wird. Zur Berechnung der Erträge werden die produktiven Zeiten I und die äquivalenten Zeiten II mit den Bruttolöhnen (einschließlich Lohnnebenkosten) von An- und Ungelernten bzw. Fachkräften bewertet (Schönfeld et al. 2010, S. 35f.). Im Durchschnitt liegen 2007 die sogenannten Äquivalenzerträge, die von den Auszubildenden am normalen betrieblichen Arbeitsplatz erwirtschaftet werden, im ersten Ausbildungsjahr bei $9.511 €$ und im dritten Jahr bei $14.592 €$. In Ostdeutschland fallen diese Erträge wesentlich niedriger aus als in Westdeutschland (Tabelle 2), was vor allem auf das unterschiedliche Lohnniveau zurückzuführen ist. Bei den Wirtschaftszweigen werden im Dienstleistungsbereich II deutlich höhere Erträge erreicht als in den anderen Bereichen. Mit zunehmender Betriebsgröße steigen die Erträge am Arbeitsplatz merklich an, ${ }^{(2)}$ dies ist jedoch allein ein Effekt der in großen Unternehmen erheblich höheren Löhne, denn die produktiven Zeiten der Auszubildenden nehmen ja umgekehrt in Großbetrieben einen geringeren Umfang

(11) Allerdings gibt es auch eine Reihe von tariflichen Vereinbarungen, die eine befristete Übernahme von Auszubildenden verbindlich vorsehen. Bei den betreffenden tarifgebundenen Betrieben kann dennoch von einem Investitionsmotiv ausgegangen werden, denn ihnen ist ja die Übernahmepflicht bewusst.

(12) Eine Ausnahme bilden die relativ niedrigen Erträge im ersten Ausbildungsjahr bei den Betrieben ab 500 Beschäftigten wegen der dort verbreiteten Lehrwerkstattausbildung. 
TABELLE 2

Produktive Leistungen am betrieblichen Arbeitsplatz im ersten und dritten Ausbildungsjahr

Durchschnittliche Angaben pro Auszubildenden im jeweiligen Ausbildungsjahr

\begin{tabular}{|c|c|c|c|c|c|c|c|c|}
\hline & \multicolumn{2}{|c|}{$\begin{array}{c}\text { Zahl produktiver Tage I } \\
\text { (Tätigkeiten An- und Ungelernter) }\end{array}$} & \multicolumn{2}{|c|}{$\begin{array}{c}\text { Zahl äquivalenter Tage II } \\
\text { (fachkraft-gleiche Tätigkeiten) }\end{array}$} & \multicolumn{2}{|c|}{$\begin{array}{l}\text { Äquivalenzerträge insgesamt } \\
\text { am Arbeitsplatz in } €\end{array}$} & \multicolumn{2}{|c|}{$\begin{array}{l}\text { Jährliche AV in \% der } \\
\text { Äquivalenzerträge am Arbeitsplatz }\end{array}$} \\
\hline & 1. AJ & 3. AJ & 1. AJ & 3. AJ & 1. AJ & 3. AJ & 1. AJ & 3. AJ \\
\hline Insgesamt & 59 & 40 & 19 & 59 & 9.511 & 14.592 & 67,5 & 54,6 \\
\hline \multicolumn{9}{|l|}{ West-Ost } \\
\hline Westdeutschland & 59 & 41 & 19 & 59 & 10.012 & 15.314 & 67,6 & 54,5 \\
\hline Ostdeutschland & 59 & 34 & 17 & 58 & 7.729 & 11.920 & 66,8 & 55,1 \\
\hline \multicolumn{9}{|l|}{ Wirtschaftszweig } \\
\hline Verarbeitendes Gewerbe ${ }^{1}$ & 58 & 41 & 14 & 51 & 9.072 & 14.720 & 69,7 & 53,6 \\
\hline Handel & 57 & 46 & 21 & 55 & 9.505 & 14.225 & 68,2 & 58,0 \\
\hline Dienstleistungen $\mathrm{I}^{2}$ & 61 & 34 & 20 & 73 & 9.461 & 14.986 & 62,3 & 49,2 \\
\hline Dienstleistungen $\mathrm{II}^{3}$ & 58 & 36 & 21 & 57 & 11.161 & 16.092 & 67,4 & 58,3 \\
\hline Verwaltung, Erziehung ${ }^{4}$ & 65 & 36 & 22 & 61 & 9.576 & 13.437 & 68,8 & 56,2 \\
\hline \multicolumn{9}{|l|}{ Betriebsgröße } \\
\hline bis 9 Beschäftigte & 67 & 43 & 19 & 68 & 8.262 & 13.015 & 65,3 & 53,7 \\
\hline 10 bis 49 Beschäftigte & 62 & 45 & 19 & 56 & 9.045 & 13.648 & 63,6 & 55,2 \\
\hline 50 bis 499 Beschäftigte & 61 & 38 & 20 & 56 & 10.945 & 15.274 & 62,1 & 54,3 \\
\hline 500 und mehr Beschäftigte & 44 & 32 & 15 & 55 & 8.548 & 16.577 & 89,2 & 55,2 \\
\hline \multicolumn{9}{|l|}{ Ausbildungsbereich } \\
\hline Industrie und Handel & 59 & 41 & 19 & 56 & 9.780 & 15.191 & 70,8 & 56,8 \\
\hline Handwerk & 60 & 39 & 16 & 54 & 8.997 & 13.559 & 60,5 & 52,1 \\
\hline Landwirtschaft & 68 & 50 & 14 & 57 & 8.977 & 13.024 & 58,7 & 51,7 \\
\hline Freie Berufe & 64 & 35 & 27 & 93 & 9.666 & 16.184 & 60,7 & 43,2 \\
\hline Öffentlicher Dienst & 53 & 29 & 14 & 42 & 8.962 & 11.375 & 89,1 & 81,1 \\
\hline \multicolumn{9}{|l|}{ Berufsart } \\
\hline kaufmännisch & 64 & 40 & 22 & 68 & 11.141 & 16.961 & 60,3 & 49,4 \\
\hline gewerblich oder technisch & 54 & 40 & 15 & 49 & 7.782 & 12.094 & 78,3 & 62,2 \\
\hline
\end{tabular}

${ }^{1}$ einschließlich Landwirtschaft;

${ }^{2}$ Gastgewerbe, Erbringung sonstiger öffentlicher und persönlicher Dienstleistungen, Verkehr und Nachrichtenübermittlung, Energie- und Wasserversorgung:

${ }^{3}$ Kredit- und Versicherungsgewerbe, Grundstücks- und Wohnungswesen, Vermietung beweglicher Sachen, Erbringung von wirtschaftlichen Dienstleistungen;

${ }_{4}^{4}$ Öffentliche Verwaltung, Verteidigung, Sozialversicherung, Erziehung und Unterricht, Gesundheits-, Veterinär- und Sozialwesen.

Quelle: BIBB-Kosten-Nutzen-Erhebung 2007

ein als in Kleinbetrieben. Zwischen den Ausbildungsbereichen unterscheiden sich die Erträge im ersten Ausbildungsjahr nur vergleichsweise gering. Im dritten Ausbildungsjahr sind sie bei den Freien Berufen überdurchschnittlich hoch, im Öffentlichen Dienst dagegen relativ niedrig.

Werden nun die Ausbildungsvergütungen in Beziehung zu den Ausbildungserträgen gesetzt, so zeigt sich Folgendes: Im ersten Ausbildungsjahr liegen die jährlichen Vergütungen durchschnittlich bei $68 \%$ der Erträge, im dritten Ausbildungsjahr erreichen sie nur $55 \%$. Zwischen West- und Ostdeutschland tritt kaum ein Unterschied bei diesen Relationen auf. Im Hinblick auf die Wirtschaftszweige fällt der Dienstleistungsbereich I mit vergleichsweise niedrigen Vergütungen in Relation zu den Erträgen auf (Tabelle 2). Nach Betriebsgröße variieren die Relationen nur relativ wenig. ${ }^{\text {(B) }}$ Verhältnismäßig große Abweichungen gibt es dagegen zwischen den Ausbildungsbereichen: Mit Abstand am höchsten ist im Öffentlichen Dienst jeweils der Anteil der Ausbildungsvergütungen an den Erträgen, am niedrigsten dagegen in der Landwirtschaft (erstes Ausbildungsjahr) bzw. bei den Freien Berufen (drittes Ausbildungsjahr).

Der Zusammenhang von Ausbildungsvergütungen und Erträgen erweist sich insgesamt gesehen in der bivariaten Analyse als nur schwach ausgeprägt. Der (jeweils signifikante) Korrelationskoeffizient nach Pearson beträgt für das erste Ausbildungsjahr 0,092 und für das dritte Ausbildungsjahr 0,207. Die bivariate Betrachtung deutet damit darauf hin, dass die (monetär bewerteten) produktiven Leistungen der Auszubildenden keinen sehr bedeutsamen Einfluss auf

(13) Wiederum ausgenommen Betriebe ab 500 Beschäftigte beim ersten Ausbildungsjahr. 
die Vergütungshöhe haben. Nun soll untersucht werden, in welcher Beziehung die von den Betrieben gezahlten Ausbildungsvergütungen zu dem betrieblichen Lohn- und Gehaltsniveau von Fachkräften sowie an- und ungelernten Arbeitskräften stehen.

\section{Vergütungen in Relation zum Lohn- und Gehaltsniveau}

In der BIBB-Kosten-Nutzen-Erhebung 2007 sind die Bruttolöhne und -gehälter, welche die Betriebe ihren Fachkräften durchschnittlich zahlen, differenziert nach kaufmännischem, technischem und gewerblichem Bereich erfasst worden. In den kaufmännischen, technischen und gewerblichen Ausbildungsberufen sind die Ausbildungsvergütungen daher in Relation zum Fachkraftentgelt im jeweils zutreffenden Bereich gesetzt worden. Demnach liegen die Ausbildungsvergütungen im ersten Ausbildungsjahr bei durchschnittlich 22 \% der Löhne bzw. Gehälter von Fachkräften. Im dritten Ausbildungsjahr werden durchschnittlich $28 \%$ des Fachkraftentgelts erreicht.

Wie Tabelle 3 zu entnehmen ist, weichen in West- und Ostdeutschland die Relationen der Ausbildungsvergütungen zu den Entgelten von Fachkräften nur wenig voneinander ab. Auch bei einer nach Wirtschaftszweigen, Betriebsgröße, Ausbildungsbereichen oder Berufsarten differenzierten Betrachtung zeigen sich nur vergleichsweise geringe Unterschiede, sie betragen maximal rund vier Prozentpunkte innerhalb des ersten bzw. dritten Ausbildungsjahres.

Wird das Verhältnis von Ausbildungsvergütungen zu den Bruttolöhnen und -gehältern von an- und ungelernten Arbeitskräften betrachtet - diese sind als betriebliche Durchschnittswerte ohne weitere Differenzierung erhoben worden -, so zeigt sich ein deutlich weniger einheitliches Bild. Im Durchschnitt beträgt die Relation im ersten Ausbildungsjahr $34 \%$ und im dritten Ausbildungsjahr $43 \%$.

In Ostdeutschland sind die Vergütungen des ersten und dritten Ausbildungsjahres im Verhältnis zu den Löhnen und Gehältern An- und Ungelernter jeweils etwas niedriger als in Westdeutschland. Bei der Unterscheidung nach Betriebsgröße ist die Relation in sehr kleinen Betrieben jeweils am höchsten, in mittleren Betrieben am niedrigsten. Im Ausbildungsbereich Öffentlicher Dienst erreichen die Vergütungen einen vergleichsweise hohen Anteil an den Entgelten An- und Ungelernter, im Handwerk dagegen einen eher niedrigen. Die beschriebenen Ergebnisse deuten auf eine relativ enge Bindung der Ausbildungsvergütungen an die Löhne bzw. Gehälter von Fachkräften hin. Der (jeweils signifikante) Korrelationskoeffizient nach Pearson beträgt für das erste Ausbildungsjahr 0,427 und für das dritte Ausbildungsjahr 0,389 , was allerdings keine besonders hohen Werte darstellt. Dies bedeutet, dass es - anders als auf den aggregierten Ebenen - auf der betrieblichen Ebene, d.h. zwischen den einzelnen Betrieben, deutliche Unterschiede in den Relationen von Ausbildungsvergütungen und Fachkraftlöhnen gibt. Die Zusammenhänge zwischen Ausbildungsvergütungen und Entgelten An- und Ungelernter sind mit einem Koeffizienten für das erste Ausbildungsjahr von 0,336 und für das dritte Ausbildungsjahr von 0,317 noch ein wenig schwächer. Ob die Löhne und Gehälter der Fachkräfte bzw. An- und Ungelernten im Zusammenspiel aller für die Höhe der Ausbildungsvergütungen möglicherweise relevanten Merkmale und Größen tatsächlich als Einflussgrößen bedeutsam sind, kann nur mit Hilfe multivariater Analysen geklärt werden. Damit befasst sich der nächste Abschnitt.

\section{Determinanten der Vergütungshöhe}

Mittels multipler linearer Regressionsmodelle lassen sich die Merkmale bzw. Größen identifizieren, die einen eigenständigen Effekt auf die Höhe der von den Betrieben gezahlten Ausbildungsvergütungen haben, denn es werden jeweils alle anderen einbezogenen möglichen Einflussfaktoren kontrolliert. Die Modelle beziehen sich zum einen auf die Ausbildungsvergütungen im ersten Ausbildungsjahr (Modell 1), zum anderen auf die Vergütungen im dritten Ausbildungsjahr (Modell 2) (Tabelle 4). Im Mittelpunkt der Analysen steht die Frage, ob sich für die Faktoren, die einerseits auf den Entgeltcharakter der Ausbildungsvergütungen (produktive Leistungen der Auszubildenden) und andererseits auf den Beihilfecharakter hinweisen (tarifrelevante Betriebsmerkmale und betriebliche Faktoren wie z.B. Wirtschaftszweig, betriebliches Lohn- und Gehaltsniveau von Fachkräften) statistisch bedeutsame Einflüsse nachweisen lassen. In Bezug auf die produktiven Leistungen werden die produktiven Tage I und die äquivalenten Tage II, also die (unbewerteten) Produktivzeiten, einbezogen. Als tarifrelevante Betriebsmerkmale werden die Strukturmerkmale Region, Wirtschaftszweig, Betriebsgröße, Ausbildungsbereich und Berufsart aufgenommen. Mit den beiden Variablen tarifliche Bindung und Betriebsrat werden betriebliche Rahmenbedingungen der Vergütungszahlungen berücksichtigt, die darauf hinweisen, ob kollektivvertragliche Regelungen für die betriebliche Ebene tatsächlich relevant werden. Als weitere Rahmenbedingung wird das aus dem Übernahmeverhalten ableitbare betriebliche Ausbildungsmotiv einbezogen. Schließlich werden als betriebliche Faktoren noch das betriebliche Lohnniveau von Fachkräften sowie von An- und Ungelernten in die Modelle eingeschlossen.

Was die tarifrelevanten Betriebsmerkmale anbetrifft, so bestätigen sich in den Regressionsmodellen weitgehend die Befunde aus den bivariaten Analysen. So zeigt sich beispielsweise in beiden Modellen, dass die Vergütungen im Osten signifikant niedriger als im Westen sind, in mittleren und großen Betrieben deutlich höher als in sehr kleinen 
Anteil der Ausbildungsvergütungen im ersten und dritten Ausbildungjahr an den Entgelten von Fachkräften bzw. An- und Ungelernten

Durchschnittliche Angaben pro Auszubildenden im jeweiligen Ausbildungsjahr

\begin{tabular}{|c|c|c|c|c|c|c|}
\hline & \multicolumn{2}{|c|}{$\begin{array}{l}\text { Ausbildungsvergütung (AV) } \\
\text { pro Monat in } €\end{array}$} & \multicolumn{2}{|c|}{$\begin{array}{c}\text { AV in \% des } \\
\text { Fachkraftentgelts }\end{array}$} & \multicolumn{2}{|c|}{$\begin{array}{l}\text { AV in \% des Entgelts An- } \\
\text { oder Ungelernter }\end{array}$} \\
\hline & 1. AJ & 3. AJ & 1. AJ & 3. AJ & 1. AJ & 3. AJ \\
\hline Insgesamt & 535 & 664 & 22,4 & 28,1 & 33,9 & 42,7 \\
\hline \multicolumn{7}{|l|}{ West-Ost } \\
\hline Westdeutschland & 564 & 695 & 22,6 & 28,2 & 34,4 & 43,0 \\
\hline Ostdeutschland & 430 & 547 & 21,7 & 27,4 & 31,6 & 41,2 \\
\hline \multicolumn{7}{|l|}{ Wirtschaftszweig } \\
\hline Verarbeitendes Gewerbe $^{1}$ & 527 & 658 & 21,1 & 26,5 & 32,0 & 40,6 \\
\hline Handel & 540 & 688 & 24,1 & 30,9 & 34,5 & 45,6 \\
\hline Dienstleistungen $\mathrm{I}^{2}$ & 491 & 615 & 22,2 & 27,7 & 32,2 & 41,1 \\
\hline Dienstleistungen $\|^{3}$ & 627 & 782 & 23,5 & 29,1 & 35,4 & 43,5 \\
\hline Verwaltung, Erziehung ${ }^{4}$ & 549 & 630 & 22,6 & 27,2 & 39,1 & 45,0 \\
\hline \multicolumn{7}{|l|}{ Betriebsgröße } \\
\hline bis 9 Beschäftigte & 450 & 583 & 22,5 & 30,3 & 37,6 & 48,4 \\
\hline 10 bis 49 Beschäftigte & 480 & 628 & 21,5 & 27,9 & 33,0 & 43,4 \\
\hline 50 bis 499 Beschäftigte & 567 & 691 & 23,1 & 27,8 & 32,4 & 39,9 \\
\hline 500 und mehr Beschäftigte & 636 & 762 & 22,3 & 26,7 & 35,2 & 42,1 \\
\hline \multicolumn{7}{|l|}{ Ausbildungsbereich } \\
\hline Industrie und Handel & 577 & 719 & 23,7 & 29,2 & 34,3 & 42,9 \\
\hline Handwerk & 453 & 588 & 19,5 & 25,4 & 30,9 & 40,6 \\
\hline Landwirtschaft & 439 & 562 & 22,2 & 28,4 & 32,0 & 41,3 \\
\hline Freie Berufe & 489 & 583 & 22,0 & 29,3 & 37,3 & 47,1 \\
\hline Öffentlicher Dienst & 666 & 768 & 24,0 & 28,5 & 44,1 & 49,1 \\
\hline \multicolumn{7}{|l|}{ Berufsart } \\
\hline kaufmännisch & 560 & 698 & 22,5 & 28,1 & 34,7 & 43,8 \\
\hline gewerblich oder technisch & 508 & 627 & 22,4 & 27,9 & 32,9 & 41,5 \\
\hline
\end{tabular}

einschließlich Landwirtschaft;

${ }^{2}$ Gastgewerbe, Erbringung sonstiger öffentlicher und persönlicher Dienstleistungen, Verkehr und Nachrichtenübermittlung, Energie- und Wasserversorgung: ${ }^{3}$ Kredit- und Versicherungsgewerbe, Grundstücks- und Wohnungswesen, Vermietung beweglicher Sachen, Erbringung von wirtschaftlichen Dienstleistungen: ${ }_{4}^{4}$ Öffentliche Verwaltung, Verteidigung, Sozialversicherung, Erziehung und Unterricht, Gesundheits-, Veterinär- und Sozialwesen.

Quelle: BIBB-Kosten-Nutzen-Erhebung 2007.

Betrieben und in den Ausbildungsbereichen Öffentlicher Dienst sowie Industrie und Handel wesentlich höher als im Handwerk. Die Ergebnisse verdeutlichen, dass die tarifrelevanten Betriebsmerkmale und damit also die tarifvertraglichen Vereinbarungen für die Höhe von Ausbildungsvergütungen eine herausragende Rolle spielen.

Im Hinblick auf die betrieblichen Rahmenbedingungen, die etwas über die Relevanz kollektivvertraglicher Regelungen für den einzelnen Betrieb aussagen, wird die erhebliche Bedeutung einer tariflichen Bindung der Betriebe für die Vergütungshöhe deutlich: So zahlen Betriebe ohne eine Tarifbindung signifikant geringere Vergütungen, und auch in Betrieben, die sich an einem Tarif orientieren, wird nicht das Vergütungsniveau tarifgebundener Betriebe erreicht. Großen Einfluss auf die Vergütungshöhe hat zudem das Vorhandensein eines Betriebsrats. In Betrieben, in denen kein Betriebsrat existiert, wird den Auszubildenden deutlich weniger bezahlt. ${ }^{(1)}$

Das Ausbildungsmotiv (Investitions- vs. Produktionsmotiv) der Betriebe, soweit es sich aus deren Übernahmeverhalten ableiten lässt, schlägt sich ebenfalls merklich in der Vergütungshöhe nieder. Bei Betrieben, die ihre Auszubildenden nur teilweise oder lediglich in Ausnahmefällen nach der Ausbildung weiterbeschäftigen wollen, ist das Ver-

(4) Da Kleinstbetriebe fast nie und Großbetriebe fast immer einen Betriebsrat haben (Kriechel et al. 2012), wurden die Regressionsmodelle auch einmal nur für mittlere Betriebe mit einer Beschäftigtenzahl zwischen zehn und 100 gerechnet. Hierbei fiel jedoch der Einfluss des Vorhandenseins eines Betriebsrats ähnlich hoch aus wie in den ausgewiesenen Gesamtmodellen. 
gütungsniveau signifikant niedriger als in Betrieben, die daran interessiert sind, ihre Auszubildenden auf Dauer als Fachkräftenachwuchs zu halten.

Das Niveau der Entgelte von an- und ungelernten Arbeitskräften hat nach den Ergebnissen der Modellberechnungen keinen eigenständigen Einfluss auf die Vergütungshöhe der Auszubildenden. Demgegenüber geht allerdings ein statistisch hochsignifikanter Effekt von dem Niveau der Fachkraftlöhne und -gehälter aus, wie beide Modelle zeigen. ${ }^{\circledR}$ Je höher die Fachkraftentgelte sind, desto höher fallen auch die Vergütungen der Auszubildenden im ersten und dritten Ausbildungsjahr aus. Damit bestätigt sich - worauf die bivariaten Analysen bereits hindeuteten - auch in den multivariaten Modellen, dass es in den Betrieben eine - wenn auch nicht sehr ausgeprägte - Koppelung der Ausbildungsvergütungen an die Löhne und Gehälter der Fachkräfte gibt. Das betriebliche Lohnniveau spielt für die Festlegung von Ausbildungsvergütungen also durchaus eine Rolle. Allerdings sind die tarifrelevanten Betriebsmerkmale und die betrieblichen Rahmenbedingungen für die Höhe der Vergütungen insgesamt wesentlich wichtiger.

Für die produktiven Leistungen der Auszubildenden lassen sich dagegen keine Einflüsse auf die Höhe der Ausbildungsvergütungen in der erwarteten Richtung nachweisen. Die Vergütungen sind in Betrieben mit einem stärkeren produktiven Einsatz der Auszubildenden nicht höher als in Betrieben, die ihre Auszubildenden in geringerem Umfang produktiv arbeiten lassen. In Modell 1 zeigt sich vielmehr für das erste Ausbildungsjahr sogar ein negativer Effekt bei den produktiven Zeiten I. Dies bedeutet, dass Betriebe umso niedrigere Vergütungen zahlen, desto stärker sie ihre Auszubildenden für einfachere Tätigkeiten, die normalerweise von an- und ungelernten Arbeitskräften erledigt werden, einsetzen. Darüber hinaus sind keine statistisch signifikanten Einflüsse der produktiven Leistungen auf die Vergütungshöhe zu verzeichnen. Aus der empirischen Analyse ergeben sich also keine Hinweise darauf, dass Ausbildungsvergütungen Entgelt für erbrachte Arbeitsleistungen darstellen. Insgesamt kommt den Ausbildungsvergütungen nach den Ergebnissen der Analyse eher der Charakter einer Ausbildungsbeihilfe zu, deren Höhe über eine relative Bedarfsbestimmung vor allem aus überbetrieblichen Gegebenheiten im Rahmen tariflicher Vereinbarungen abgeleitet wird, aber auch von deren Geltung für den Betrieb abhängt und zudem von dem betrieblichen Lohnniveau mit bestimmt wird. Eine Rolle spielen darüber hinaus die betrieblichen Ausbildungsmotive.

(15) Zwischen Löhnen und Gehältern von Fachkräften sowie Anund Ungelernten besteht zwar eine signifikante Korrelation, die allerdings mit einem Koeffizienten nach Pearson von 0,269 (Betriebe mit 1. Ausbildungsjahr) bzw. 0,317 (Be triebe mit 3. Ausbildungsjahr) nicht sehr hoch ausfällt. Bei beiden Regressionsmodellen ergab sich für beide Variablen jeweils ein Varianzinflationsfaktor (VIF) von $<1,4$ und somit kein Verdacht auf Multikollinearität.

\section{TABELLE 4}

Einflüsse auf die Höhe der Ausbildungsvergütungen im ersten und dritten Ausbildungsjahr

Multiple lineare Regressionsmodelle

\begin{tabular}{|c|c|c|}
\hline & Modell 1 & Modell 2 \\
\hline Einflussgrößen & $1 . \mathrm{AJ}$ & $3 . \mathrm{JJ}$
\end{tabular}

Produktive Leistungen der Auszubildenden produktive Tage I am Arbeitsplatz äquivalente Tage II am Arbeitsplatz $-, 092$ Tarifrelevante Betriebsmerkmale

Region (Ref.: Westdeutschland)

Ostdeutschland

Wirtschaftszweig (Ref.: Verarbeitendes Gewerbe)

Handel

Dienstleistungen I

Dienstleistungen II

Verwaltung, Erziehung

Betriebsgröße (Ref.: bis 9 Beschäftigte)

10 bis 49 Beschäftigte

50 bis 499 Beschäftigte

500 und mehr Beschäftigte

Ausbildungsbereich (Ref.: Handwerk)

Industrie und Handel

Landwirtschaft

Freie Berufe

Öffentlicher Dienst

$180 *$

$-, 180$

, 049

Berufsart (Ref.: kaufmännisch)

gewerblich oder technisch

$-108,479 * * *$

$-128,289 * * *$

Betriebliche Faktoren

a) Rahmenbedingungen der Vergütungszah/ungen

tarifliche Bindung (Ref.: ja, tarifliche Bindung)

nein, aber mit Anlehnung an Tarifvertrag

nein, kein Bezug zu Tarifvertrag

Betriebsrat (Ref.: mit Betriebsrat)

ohne Betriebsrat

Übernahmeverhalten (Ref.: auf jeden Fall Übernahme)

teilweise Übernahme

nur in Ausnahmefällen Übernahme

b) Lohn- und Gehaltsniveau

Entgelt von Fachkräften

Entgelt von An- und Ungelernten

$-4,039$

$-20,041 *$

$-26,120 * *$

$-14,176$

$34,795 * * *$

$38,170 * *$

$-10,973$

$-36,282 * *$

Konstante

$13,683^{+}$

$21,927^{*}$

28,779 **

29,300 *

$46,161 * * *$

46,682 **

Fallzahl n

$88,410 * * *$

$58,932 * * *$

21,053

1,451

34,056 *

6,526

$105,167 * * *$

$78,113 * * *$

$-25,783^{* *}$

$\mathrm{R}^{2}$

Signifikanzniveau : ${ }^{+}<0,1 \quad * \mathrm{p}<0,05 \quad{ }^{* *} \mathrm{p}<0,01 \quad * * * \mathrm{p}<0,001$.

Quelle: BIBB-Kosten-Nutzen-Erhebung 2007.

\begin{tabular}{|c|c|}
\hline$-12,666^{+}$ & $-18,419 *$ \\
\hline$-40,273^{* * *}$ & $-53,513 * * *$ \\
\hline$-73,413^{* * *}$ & $-66,938 * * *$ \\
\hline$-37,323^{* * *}$ & $-25,073 * * *$ \\
\hline$-26,565^{* *}$ & $-35,329 * * *$ \\
\hline, $022 * * *$ &, $021 * * *$ \\
\hline ,007 & ,004 \\
\hline $688,451 * * *$ & $857,626 * * *$ \\
\hline 1.597 & 1.711 \\
\hline ,546 & ,447 \\
\hline
\end{tabular}




\section{Zusammenfassung und Schlussfolgerungen}

In diesem Beitrag wurde in einer empirischen Analyse die Frage untersucht, ob die Ausbildungsvergütungen in Deutschland eher als Entgelt für erbrachte Arbeitsleistungen oder als Ausbildungsbeihilfe aufgefasst werden können. Hierzu wurde der Zusammenhang zwischen der Höhe der Vergütungen und einzelnen möglichen Einflussgrößen analysiert. Es zeigt sich, dass die Festlegung von Ausbildungsvergütungen in starkem Maße durch überbetriebliche Faktoren der Tarifbildung bestimmt wird. Betriebliche Rahmenbedingungen, die insbesondere abbilden, ob tarifliche Regelungen tatsächlich Anwendung in einem Betrieb finden, haben ebenfalls eine große Bedeutung. Das innerbetriebliche Lohnniveau hat darüber hinaus auch einen wenn auch geringeren - Einfluss.

Die von den Auszubildenden während der Ausbildung erbrachten produktiven Leistungen haben indessen für die Festlegung der Höhe der Vergütungen keine Bedeutung. Insofern haben die Ausbildungsvergütungen in Deutschland - empirisch gesehen - weniger den Charakter eines Arbeitsentgelts, sondern sind eher als Ausbildungsbeihilfen anzusehen. Die Vergütungen passen also eher zum Ausbildungscharakter der betrieblichen Berufsausbildung und weniger zum Beschäftigungscharakter. Die rechtlich eigentlich gebotene Berücksichtigung der Entlohnungsfunktion von Ausbildungsvergütungen lässt sich empirisch nicht nachweisen. Die Angemessenheit der Höhe der Vergütung richtet sich in der bestehenden Praxis aber nicht nach einem absoluten Wert, sondern wird als relative Größe für einen bestimmten Tarifbereich definiert. Hierbei scheint das durchschnittliche Lohn- und Gehaltsniveau der Fachkräfte in einem Bereich als die wesentliche Orientierungsgröße zu dienen. So ergeben sich für verschiedene Wirtschaftszweige und Ausbildungsbereiche ähnlich hohe Relationen von Ausbildungsvergütungen und Fachkraftlöhnen und -gehältern.

Die große Relevanz überbetrieblicher Faktoren bei der Festlegung der Höhe der Ausbildungsvergütungen bedeutet, dass betriebliche Besonderheiten nur eine relativ geringe Rolle spielen. Insbesondere Unterschiede im Lohnniveau der Fachkräfte zwischen einzelnen Betrieben wirken sich nur in eher geringem Maße auf die Höhe der Vergütungen aus. Die Relationen von Ausbildungsvergütungen und Fachkraftlöhnen können sich somit zwischen den einzelnen Betrieben deutlich unterscheiden. Geht man davon aus, dass die Differenz zwischen Vergütung und Fachkraftlohn auch ein Bestimmungsfaktor der betrieblichen Ausbildungsbereitschaft ist, so lässt sich Folgendes schlussfolgern: Bei einer überbetrieblich fixierten Vergütungshöhe sind für Betriebe mit einem niedrigeren Lohnniveau die ökonomischen Anreize für eine Ausbildung geringer und manche Betriebe (Grenzanbieter) werden sicherlich auch von einer Ausbildung abgehalten. Unter Gesichtspunkten der Aufrechterhaltung einer Mindestqualität der betrieblichen Ausbildung kann dies aber durchaus vorteilhaft sein. Denn gerade bei Betrieben mit einem Lohnniveau weit unterhalb des Branchendurchschnitts und dann auch sehr niedrigen Ausbildungsvergütungen bestünde die Gefahr, dass attraktive Ausbildungsbedingungen kaum geschaffen und insofern auch keine zukunftsträchtigen Perspektiven für den Nachwuchs eröffnet würden.

Der nicht bestehende Zusammenhang zwischen Ausbildungsvergütungen und produktiven Leistungen der Auszubildenden impliziert, dass Auszubildende keine Verbindung zwischen ihrem erbrachten Produktivbeitrag und ihrer Vergütung erleben. Betriebliche Ausbildung ist aber durchaus auch bereits Arbeit und insofern könnte eine unzureichende Verbindung von Arbeitsleistung und Entlohnung, als die die Vergütung auch zu sehen ist, für die Auszubildenden subjektiv ein Problem darstellen. So geht aus einer empirischen Studie des Bundesinstituts für Berufsbildung hervor, dass Auszubildende mit sehr hohen Produktivbeiträgen in der Ausbildung deutlich unzufriedener mit ihrer Ausbildungsvergütung sind (Beicht/Krewerth 2010, S. 12f.). Eine solche Unzufriedenheit könnte sich auch negativ auf die Ausbildungsmotivation auswirken. Insofern wäre in Branchen mit besonders hohen Produktivbeiträgen der Auszubildenden zu überlegen, ob bei der Festlegung der Vergütungshöhe der Gesichtspunkt erbrachter produktiver Leistungen nicht stärker berücksichtigt werden sollte. Vor dem Hintergrund der sich aufgrund der demografischen Entwicklung radikal verändernder Rahmenbedingungen auf dem Ausbildungsstellenmarkt und den sich verschlechternden Möglichkeiten der Nachwuchsrekrutierung insbesondere in einzelnen Branchen und Berufen wird künftig ohnehin über die Vergütungshöhe als ein Instrument zur Gewinnung von jungen Menschen nachgedacht werden. Allerdings besteht hier auch die Gefahr, dass die höheren Vergütungen von den Jugendlichen fälschlicherweise als ein Signal für höhere Fachkraftlöhne verstanden würden. 


\section{LITERATUR}

Beicht, U. (2011): Langzeitentwicklung der tariflichen Ausbildungsvergütung in Deutschland, Bonn

Beicht, U./Krewerth, A. (2010): Geld spielt eine Rolle! Sind Auszubildende mit ihrer Vergütung zufrieden?, Bielefeld

Bispinck, R./Schulten, Th. (2009): Re-Stabilisierung des deutschen Flächentarifvertragssystems, in: WSI-Mitteilungen 62 (4), S. $201-209$,

http://www.boeckler.de/wsimit_2009_04_bispinck_schulten.pdf

Bundesinstitut für Berufsbildung (BIBB) (2012): Tarifliche Ausbildungsvergütungen 2011: Azubi-Gehälter legen deutlich zu, Pressemitteilung 01, Bonn Deutscher Bundestag (1969): Schriftlicher Bericht des Ausschusses für Arbeit zum Entwurf des im Jahre 1969 verabschiedeten Berufsbildungsgesetzes,

Deutscher Bundestag, 5. Wahlperiode, Drucksache V/4260, Bonn

Ellguth, P./Kohaut, S. (2008): Tarifbindung und betriebliche Interessenvertretung: Aktuelle Ergebnisse aus dem IAB-Betriebspanel 2007, in: WSI-Mitteilungen 61 (9), S. 515-519, http://www.boeckler.de/wsimit_2008_09_ellguth.pdf Ellguth, P./Kohaut, S. (2011): Tarifbindung und betriebliche Interessenvertretung: Aktuelle Ergebnisse aus dem IAB-Betriebspanel 2010, in: WSI-Mitteilungen 64 (5), S. 243-247, http://www.boeckler.de/wsimit_2011_05_ellguth.pdf Hans-Böckler-Stiftung (Hrsg.) (2012): Ausbildungsvergütungen zwischen 332 und 1.379 Euro, WSI-Tarifarchiv: Regionale Unterschiede bis zu $388 €$ im Monat, Pressemitteilung, 24.04., Düsseldorf

Herkert, J./Töltl, H. (2010): Berufsbildungsgesetz. Kommentar mit Nebenbestimmungen, Regensburg

Kriechel, B./Muehlemann, S./Pfeifer, H./Schuette, M. (2012): Works Councils, Collective Bargaining and Apprenticeship Training, IZA Discussion Paper (6497), Bonn

Lindley, R. M. (1975): The Demand for Apprentice Recruits by the Engineering Industry, 1951-71, in: Scottish Journal of Political Economy 22 (1), S. 1-24 Merrilees, W. J. (1983): Alternative Models of Apprentice Recruitment: With Special Reference to the British Engineering Industry, in: Applied Economics 15 (1), S. 1-21
Schnabel, C. (2005): Gewerkschaften und Arbeitgeberverbände. Organisationsgrade, Tarifbindung und Einflüsse auf Löhne und Beschäftigung, in: Zeitschrift für ArbeitsmarktForschung 38 (2/3), S. 181-196

Schönfeld, G./Wenzelmann, F./Dionisius, R./Pfeifer, H./Walden, G. (2010):

Kosten und Nutzen der dualen Ausbildung aus Sicht der Betriebe. Ergebnisse der vierten BIBB-Kosten-Nutzen-Erhebung, Bielefeld

Wenzelmann, F. (2012): Ausbildungsmotive und die Zeitaufteilung der Auszubildenden im Betrieb, in: Journal for Labour Market Research, Online First $25 \mathrm{~S}$.

\section{AUTOREN}

URSULA BEICHT ist Mitarbeiterin im Arbeitsbereich Berufsbildungsangebot und -nachfrage/Bildungsbeteiligung im Bundesinstitut für Berufsbildung (BIBB). Arbeitsschwerpunkte: Bildungsbiografie von Jugendlichen und jungen Erwachsenen, Übergang Schule - Berufsausbildung, Ausbildungsvergütungen

beicht@bibb.de

GÜNTER WALDEN, Dr., ist Abteilungsleiter der Abteilung Sozialwissenschaftliche Grundlagen der Berufsbildung im BIBB. Arbeitsschwerpunkte: Kosten und Nutzen der beruflichen Bildung.

walden@bibb.de 